\title{
Review of Packet Scheduling Algorithms in Mobile Ad Hoc Networks
}

\author{
C.Annadurai \\ Assistant Professor/ECE, SSN College of Engineering, \\ Kalavakkam - 603 110, Chennai. \\ Tamilnadu, India
}

\begin{abstract}
Scheduling algorithms are important components in the provision of guaranteed quality of service parameters. The design of scheduling algorithms for mobile ad hoc networks is challenging one because of highly variable link error rates and dynamic nature of the network. This paper provides a survey of scheduling techniques in wireless Ad hoc networks. Desirable features and classifications of schedulers for the different scheduling algorithms are discussed.
\end{abstract}

Keywords: Scheduling, algorithms, Ad hoc networks.

\section{INTRODUCTION}

An ad hoc network is a self-organizing wireless network comprised only of mobile nodes interconnected by a multihop path and without the support of any pre-existing wired infrastructure. In these networks, the mobility of nodes and the error-prone nature of the wireless medium introduce many challenges, including frequent route changes and packet losses. These problems increase the packet delays and decrease the throughput. As traffic load in the network increases, the performance of the network decreased [1]. Research in this area has focused primarily on routing protocols and medium access control (MAC). However, there is little understanding of the queuing dynamics in the nodes of these networks and different packet scheduling algorithms in the queues of the nodes provide solution for performance degradation. Scheduling algorithm manages the changes in queuing dynamics in different situation also improves the performance of the network.

This paper begin by describe the packet scheduler classification. Section III describes importance of fair scheduling and also reviews the different fair scheduling algorithms. Section IV describes the QoS aware scheduling algorithms. Different types of Priority scheduling were discussed in section V. Section VI handles the Opportunistic packet scheduling. Section VII deals different message scheduling. Finally concludes this paper.

\section{SCHEDULER CLASSIFICATION}

The schedulers are broadly classified as centralized and distributed schedulers. The distributed schedulers are suitable for ad hoc network. The Schedulers can also be classified as work-conserving or non-work-conserving [1]. A workconserving scheduler is never idle if there is a packet awaiting transmission. Examples include Generalized Processor Sharing (GPS), packet-by-packet GPS also known as Weighted Fair Queuing (WFQ), Virtual Clock (VC), Weighted Round-Robin (WRR), Self-Clocked Fair Queuing (SCFQ), and Deficit Round-Robin (DRR). In contrast, a nonwork-conserving scheduler may be idle even if there is a backlogged packet in the system because it may be expecting another higher-priority packet to arrive. Examples are Hierarchical Round-Robin (HRR), Stop-and-Go Queuing (SGQ), and Jitter-Earliest-Due-Date (Jitter- EDD). Non-workconserving schedulers generally has higher average packet delay than their work-conserving counterparts but may be used in applications where time jitter is more important than delay. In that some of the schedulers are originally proposed for wireline networks. These algorithms can be used as the error-free service model in the design of wireless schedulers. The figure 1 shows the classification of scheduling algorithms on different criteria.

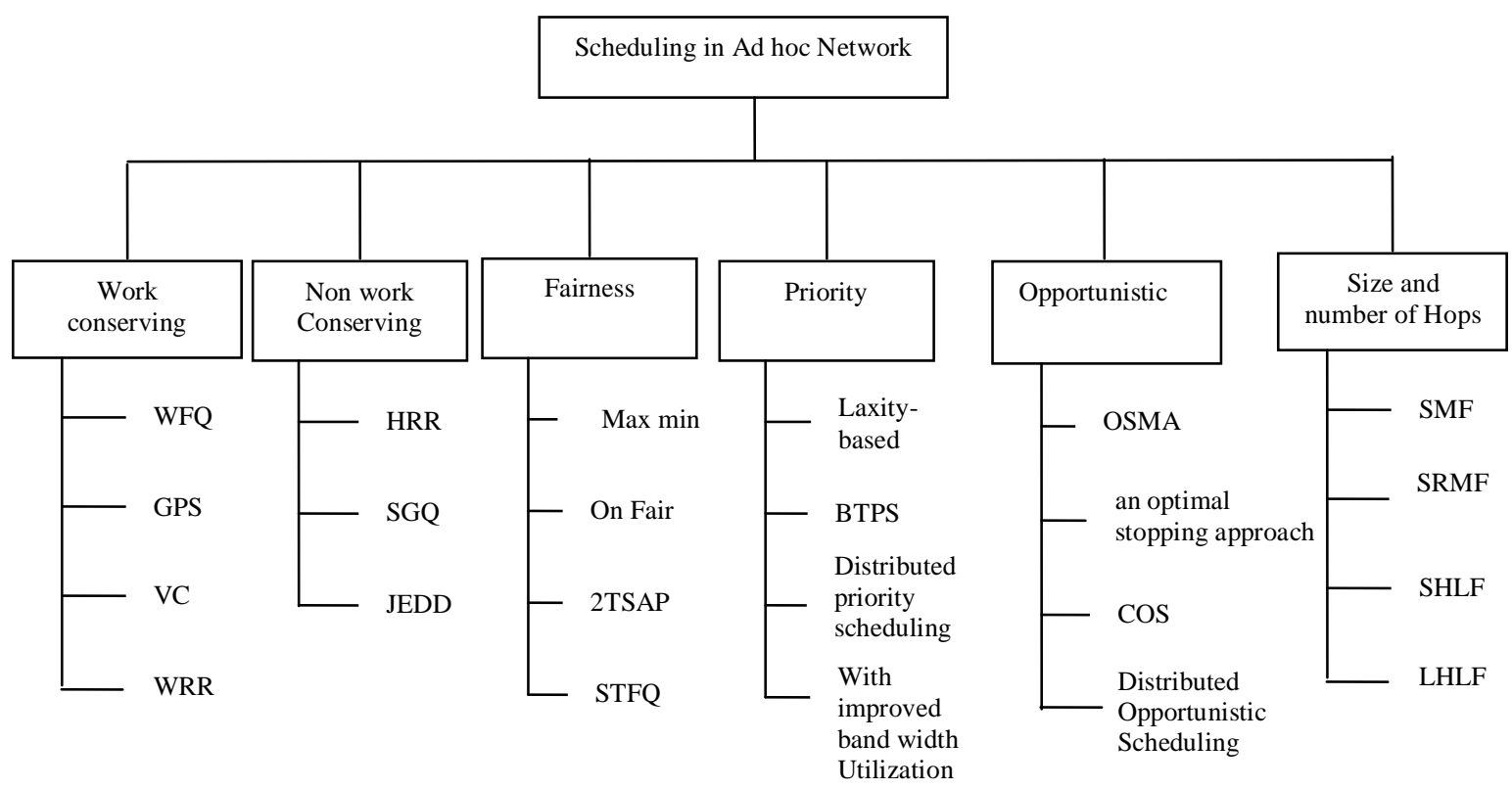

Figure 1 Classification of scheduling algorithms in Ad hoc Network 


\section{FAIR SCHEDULING}

To determine the characteristics of a suitable scheduling algorithm, consider the requirements of some of the principal applications envisioned for integrated services networks.

- Audio Applications: To maintain adequate interactivity for such applications, scheduling algorithms must provide low average and maximum delay.

- Video Applications: Variable bit rate (VBR) video sources, which are expected to impose significant requirements on network resources, have unpredictable as well as highly variable bit rate requirement at multiple time-scales.

These features impose two key requirements on network resource management. Due to the difficulty in predicting the bit rate requirement of VBR video sources, video channels may utilize more than the reserved bandwidth. Fair scheduling in ad hoc networks can be classified into two categories: timestamp based and credit based. According to timestamp based mechanism packet is locally assigned two timestamps: a start tag and a finish tag. Either timestamp can be chosen as the service tag. The packet with the smallest service tag will be sent first. In credit based scheduling is based on the unused credit can be accumulated for future use. Each flow is associated with three parameters: a credit, a usage, and an excess, where excess = usage - credit. The one with the smallest excess value has the priority to transmit.

\subsection{Start-time Fair Queueing}

In Start-time Fair Queueing algorithm (SFQ), two tags namely a start tag and a finish tag is associated with each packet. However, unlike WFQ and SCFQ, packets are scheduled in the increasing order of the start tags of the packets. Furthermore, is defined as the start tag of the packet in service at time. Where as in SFQ, on arrival, a packet is stamped with start tag and finish tag of packet were computed and tag with the packet depending upon the weight of the flow. Packets are serviced in the increasing order of the start tags, ties are broken arbitrarily. The computation of in SFQ is inexpensive since it only involves examining the start tag of packet in service.

\subsection{Maximize-local-minimum Fair Queueing}

In this algorithm, each node is responsible for assigning tags and scheduling flows that originate from it. At the same time, it also maintains a table that records service tags for other flows in its one-hop neighborhood. Start-time Fair-queueing (SFQ) used to assign start tags and finish tags for the flows because of its ease in virtual time maintenance. In each table entry, record the [flowid, flowtag], where the flowtag is the most recent service tag that the node is updated for flow id. This algorithm based on the following two mechanisms are Maximizing local minimum by transmitting flows with local minimum service tags and backoff mechanism to increase spatial reuse.

\subsection{Timestamp-Based Compensation Protocol (TBCP)}

Timestamp-Based Compensation Protocol (TBCP) is for multihop wireless networks, under which channel errors are considered [5]. TBCP adopts Start-time Fair Queueing (SFQ) as its scheduling discipline and selects the start tag as its service tag. In TBCP, the transmission order of a packet is determined with the service tag of the packet, the number of slots per frame a flow can use, and flow's Q-size. Then, each node exchanges the information about the transmission order of packets with its neighbors. Thus each node knows the service tags of other nodes, and also learns when it will transmit packets. Each node keeps monitoring its channel state. When the channel is error-prone, the node stops exchanging transmission messages with its neighbors. Once the channel recovers, the error-prone node resumes the exchanges. Consequently, if this node has packets with service tags smaller than its neighbors after recovery, these packets still have higher priority to be transmitted. Multihop flows are also handled by TBCP with introducing new parameter called Q size.

\subsection{Maxmin Fair Scheduling}

The algorithm describes that for the special case that every session always has a packet to transmit [6]. Each node allocates service tokens to the sessions traversing the node in a round-robin-like fashion. Weight of an edge in the topology graph in a slot is the minimum of the number of tokens of the corresponding session at the session's source and destination. In each slot, the sessions that constitute a maximum weighted matching in the topology graph are scheduled for service. Whenever a session is served, a token is removed from both its source and destination.

\subsection{Two-Tier Slot Allocation Protocol}

This algorithm has cluster-based mechanism. Each cluster has a scheduler (or master). Clusters may be overlapped, each with a designated code. The cluster can be formed as follows. Each mobile node periodically broadcasts beacons. Based on the received beacons, mobile nodes learn their neighbors, and related information, such as node ID, node stability. According to the selected criterion, each cluster can determine its scheduler. To receive the service, each node must join a cluster and register with the scheduler. Each scheduler periodically advertises itself as the scheduler to its cluster, from which newly arriving mobile nodes learn where to register.

\section{PRIORITY SCHEDULERS}

In priority scheduling, among all the nodes some of the nodes get higher priority than other nodes to transmit the packets from its queues. The priority scheduling helps to achieve a fairness and QoS parameters. In [16], priority scheduling in multi-hop networks was implemented. In BTPS scheme, using two narrow-band busy tone signals to ensure medium access for high priority source stations. The two narrow-band busy tone signals named BT1 and BT2. Low priority source stations determine the presence of high priority packets by sensing the carrier on the busy tone channels. The difference between IEEE 802.11 Distributed Coordination Function (DCF) and BTPS is that high priority and low priority source stations behave differently during "IFS" and "backoff" stages in BTPS.

In [17], current packet delivery ratio of a flow for which an entry is present in the node's scheduling table (ST), is an important parameter used for calculating the priority of a packet belonging to that flow with respect to packets belonging to other flows that have entries in the ST of the node. Hence, a node needs to keep track of the packet delivery ratios of all flows that have entries in its ST. This is achieved by means of a feedback mechanism. Incoming packets to a node are first queued in the node's input queue according to their arrival times. The scheduler sorts them according to their priority values and inserts them into the transmission queue. The highest priority packet is selected from the transmission queue and transmitted. The node, after transmitting a packet, 
updates the information maintained in its packet delivery ratio table (PDT) regarding the number of packets transmitted so far. The destination node of a flow on receiving data packets initiates a feedback by means of which information about the number of packets received by it is conveyed to the source. The priority indices of packets form the basis for scheduling the next packet for transmission.

In [18], scheme is to perform a priority scheduling in wireless ad-hoc networks, with full utilization of the bandwidth available, mitigating the loss of packets incurred by the mobility of the nodes in the network. Here, two narrow busy tone packets are used to indicate the notification of a transmission by a high priority node, thus resolving the contention of the channel. The Piggybacking scheme exploits the channel dynamics and its basic idea is to let the high priority traffic help the low priority traffic by sharing unused residual bandwidth.

In [19], two mechanisms are used for QoS communication in multi-hop wireless networks. First, one is distributed priority scheduling, a technique that piggybacks the priority tag of a node's head-of-line packet onto handshake and data packets; e.g., RTS/DATA packets in IEEE 802.11. By monitoring transmitted packets, each node maintains a scheduling table which is used to assess the node's priority level relative to other nodes. Then incorporate this scheduling table into existing IEEE 802.11 priority back-off schemes to approximate the idealized schedule. Second, a scheduling scheme termed multi-hop coordination so that downstream nodes can increase a packet's relative priority to make up for excessive delays incurred upstream.

\section{OPPORTUNISTIC PACKET SCHEDULING}

In Opportunistic packet Scheduling and Media Access control (OSMA) protocol [20], solving the Head-of-Line (HOL) blocking problem and the overall system throughput increased. The key mechanisms of OSMA protocol are multicast RTS and priority-based CTS. In the OSMA protocol, RTS includes a list of candidate receivers. Among those who are qualified to receive data, the one with the highest order would be granted to catch the channel by replying CTS in the first place. The ordering list will be updated dynamically according to certain scheduling policy such as Round Robin (RR) and Earlier timestamp First (ETF), so other performance metrics, can be enhanced.

In [21], discuss the link layer scheduling problem in wireless ad hoc networks. In such a network, the communication links compete for the scarce and time-varying wireless channels. Distributed Cooperative and Opportunistic Scheduling (COS) algorithm realizes the optimal scheduling policies by introducing the cooperation among neighboring transmitters. The local contention graph (LCG) is formulated with in the two hop region. The average LCG can be built with the help the FIOID scheme. By inserting extra intervals into consecutive data transmissions of the unscheduled transmitters, the scheduled links would be associated with higher priority to access the wireless channels.

In [22], consider a distributed opportunistic scheduling (DOS) in wireless ad-hoc networks, where many links contend for the same channel using random access. In such networks, distributed opportunistic scheduling involves a process of joint channel probing and distributed scheduling. Due to channel fading, the link condition corresponding to a successful channel probing could be either good or poor. In the latter case, further channel probing, although at the cost of additional delay, may lead to better channel conditions and hence higher transmission rates. The desired tradeoff boils down to judiciously choosing the optimal stopping strategy for channel probing and the rate threshold.

In [14], algorithm concentrates on error compensation, link status estimation and fair scheduling into consideration simultaneously for distributed scheduling in multihop ad hoc networks. It has a framework, named "Robust Opportunistic Scheduling for Ad Hoc Networks" (ROSA), with which a scheduling algorithm originally designed for infrastructured wireless networks can be adapted to multihop ad hoc networks. The adapted algorithm performs distributed scheduling opportunistically by utilizing the link status information provided by ROSA. This mechanism also improves the robustness of the system by limiting the traffic load at the MAC layer.

\section{SIZE AND NUMBER OF HOPS}

This section deals about the different scheduling algorithms assign priorities to the messages depending upon its size and number of hops. In Smallest Message First (SMF), the packets are scheduled in ascending order of the size of the messages of which they are a part. Packets belonging to smaller messages receive higher priority over packets belonging to larger messages. In order to implement this scheme, the total message size must be attached to each packet at the time of message fragmentation, and can be done very easily. Each router then checks the message size of each packet so that they can be queued.

In Smallest Remaining Message (SRMF) scheme [23, 24], packets are ordered on the basis of the amount of message still remaining to be sent after the current packet. This requires having additional information in the packet in the form of sequence numbers, along with the total length of the message. It is slightly more complicated than including just the total message length, and can be easily implemented. This scheme performs better than the Smallest Message First scheme.

In Shortest Hop-Length First (SHLF) scheduling[23, 24], the distance between the source and the destination, as measured by the number of hops, influences the time a packet takes to reach the destination. This strategy also belongs to the class of Head of Line queuing algorithms, and results in decreasing end-to-end packet delay of the network. The scheduling decision is made at each node independently and is based on the distance to the destination. This requires that each packet carry the total number of hops to the destination.

Longest Hop-length First (LHLF) scheduling, the metric used is the number of hops the packet requires to reach its destination. Packets having to traverse over a long number of hops will have a long end-to-end delay and transmission delay. To reduce these delays in this scheme the assign higher priority to higher priority to packets that have to higher priority to packets that have to travel over a larger number of hops.

\section{CONCLUSIONS}

In this paper, a survey of schedulers for Wireless Ad hoc networks was provided. Desirable features and design challenges and classification of such schedulers were outlined for wireless Ad hoc networks. The emergence of new multimedia and Internet applications for the wireless domain has insisted to study the scheduling algorithms for providing QoS guarantees. These guarantees are usually in the form of 
bounded delay and jitter, guaranteed rate and fairness among sessions.

\section{REFERENCES}

[1] Hossam Fattah and Fyril Leung, An Overview of Scheduling Algorithms in Wireless Multimedia Networks IEEE Wireless Communications, October 2002

[2] Byung-Gon Chun, Mary Baker, Evaluation of Packet Scheduling Algorithms in Mobile Ad hocNetworks, Mobile Computing and Communications Review, Volume 1, Number 2

[3] Vishnu, M, Mark, J.W, HOL-EDD: a flexible service scheduling scheme for ATM networks, INFOCOM '96, Proceedings IEEE Volume 2, 24-28 March 1996 Page(s):647 654 vol.2

[4] R. Srikant, Fair Resource Allocation in Wireless Networks Using Queue- Length-Based Scheduling and congestion Control, IEEE/ACM TRANSACTIONS ON NETWORKING, VOL. 15, NO. 6, DEC 2007.

[5] Hsi-Lu Chao, and Wanjiun Liao, Fair Scheduling in Mobile Ad Hoc Networks With Channel Errors, IEEE TRANSACTIONS ON WIRELESS COMMUNICATIONS, VOL. 4, NO. 3, MAY 2005

[6] Leandros Tassiulas, and Saswati Sarkar, Maxmin Fair Scheduling in Wireless Ad Hoc Networks, IEEE JOURNAL ON SELECTED AREAS IN COMMUNICATIONS, VOL. 23, NO. 1, JANUARY 2005

[7] Pawan Goyal, Harrick M. Vin, and Haichen Cheng, Start-Time Fair Queueing: A Scheduling Algorithm for Integrated Services Packet Switching Networks, IEEE/ACM TRANSACTIONS ON NETWORKING, VOL. 5, NO. 5, OCTOBER 1997

[8] Hsi-Lu Chao, Jia-Chun Kuo and Wanjiun Liao Fair Scheduling with QoS Support in Ad hoc Networks, Proceedings of the 27th Annual IEEE Conference on Local Computer Networks (LCN.02).

[9] Hsi-Lu Chao, Hsinchu,On Fair Scheduling for Mobile Ad Hoc Networks with Channel Errors, IEEE TRASACTIONS ON WIRELESS COMMUNICATIONS, VOL.4. NO.3, MAY,2005

[10] Pawan Goyal, Harrick M. Vin, and Haichen Cheng StartTime Fair Queueing: A Scheduling algorithm for Integrated Services Packet Switching Networks IEEE/ACM Transactions on Networking, vol. 5, no.5, October 1997.

[11] Qui Chen, Quian Zhang, Zhisheng Niu, QoS-Aware Cooperative and Opportunistic Scheduling Exploiting Multiuser Diversity for Rate-Adaptive Ad Hoc Networks, IEEE TRANSACTIONS ON Vehicular Technology, VOL. 57, NO. 2, March 2008.
[12] Haiyun Luo, Jerry Cheng, Songwu Lu, Self-coordinating Localized Fair Queueing in Wireless Ad Hoc Networks, IEEE Transactions on Mobile Computing, Vol. 3, No. 1, January-March 2004.

[13] Haiyun Luo and Songwu Lu, "A topology-independent Fair Queueing model in ad hoc wireless Networks," Proc IEEE ICNP'00, pp. 325-335.

[14] Yijiang Sun, Victor O.K. Li and Ka-Cheong Leung, Distributed Opportunistic Scheduling in Multihop wireless Ad Hoc Networks, IEEE, 2008

[15] Brahma, K. W. Kim, M. EL Hachimi, A. Abouaissa, P. Lorenz, "A Buffer and Energy Based Scheduling in Mobile Ad hoc Networks over Link Layer", Proceedings of the Advanced International Conference on Telecommunications and International Conference.

[16] XUE YANG and NITIN VAIDYA, Priority Scheduling in Wireless Ad Hoc Networks, Springer, Wireless Networks 12, 273-286, 2006.

[17] Karthigeyan, B. S. Manojand C. Siva Ram Murthy, A distributed laxity-based priority scheduling scheme for time-sensitive traffic in mobile ad hoc networks, Elsevier, Ad Hoc Networks Volume 3, Issue 1, January 2005, Pages 27-50

[18] Gunasekaran.R, Rhymend Uthariaraj.V, Rajesh.R, Kaarthikeyan.S, Aravind.S, Priority scheduling in mobile ad hoc networks with improved bandwidth utilization, CCECE 2008, Canadian Conference on Volume, Issue, 4-7 May 2008

[19] V.Kanodia, C.Li, Distributed Priority Scheduling and Medium Access in Ad-hoc Networks, ACM Wireless Networks, Volume 8, and November 1, 2002

[20] Jianfeng Wang, Hongqiang Zhai and Yuguang Fang, Opportunistic Packet Scheduling and Media Access Control for Wireless LANs and Multi-hop Ad Hoc Networks, IEEE Communications Society, WCNC 2004.

[21] Qing Chen, Qian Zhang, Zhisheng Niu, Opportunistic Link Scheduling with QoS Requirements in Wireless Ad Hoc Networks, IEEE Communications Society subject matter experts for publication in the ICC 2007 proceedings.

[22] Dong Zheng, Weiyan Ge and Junshan Zhang, Distributed Opportunistic Scheduling For Ad-Hoc Communications: An Optimal Stopping Approach, MobiHoc'07, September 9-14, 2007 ACM.

[23] Ravikiran Kakaraparthi, Ramnath Duggirala and Dharma P. Agrawal, Efficient Message Scheduling in Ad hoc Networks, IEEE 2000.

[24] Eytan Modiano. Scheduling packet transmissions in a multi-hop packet switched network based on message length. In Proceedings of the Sixth International Conference on Computer Communications and Networks, pages 350-357, 1997. 